\title{
Insights into the effects of inducible and neuronal nitric oxide synthase isoenzymes in experimental intestinal heterophyiasis \\ Original
}

Article

Marwa O Abdel Aziz ${ }^{1}$, Heba O Elsayed ${ }^{2}$

Department of Medical Parasitology, Faculty of Medicine, Zagazig University, Zagazig, 44519, Egypt ${ }^{1}$, LIS: Cross-National Data Center, Maison des Sciences Humaines - 5e étage, 11- porte des Sciences, L-4366 Esch-Belval, Luxembourg ${ }^{2}$

\begin{abstract}
Background: Heterophyiasis is one of the food-borne trematode infections, caused by the intestinal fluke Heterophyes heterophyes. The exact role of nitric oxide (NO) in the immune response against the majority of parasites remains controversial. It proved protective against a wide range of protozoan and helminthic parasites. Nevertheless, its role in intestinal heterophyiasis is yet to be explored.

Objectives: The study aims to explore the possible roles of inducible nitric oxide synthase (iNOS) and neuronal nitric oxide synthase (nNOS) in experimental intestinal heterophyiasis.

Material and Methods: The experimental study design included infection of male puppies with $H$. heterophyes encysted metacercariae (EMC), followed by treatment with aminoguanidine (AG) and 7-nitroindazole (7-NI) drugs, as selective inhibitors of iNOS and nNOS, respectively. Controls included non-infected and infected untreated puppies. Intestinal tissue sections from all puppies were stained for histopathological and immunohistochemical (IHC) assessments.

Results: Different intensities of iNOS and nNOS isoenzymes were observed in intestinal sections. The study showed the highest concentration of iNOS isoenzyme in the infected-7-NI treated group. The control noninfected puppies exhibited the highest levels of nNOS expression, with statistical significance $(P<0.05)$. The study also showed that AG significantly reduced the degree of inflammatory cellular infiltrations. Additionally, the over-production of NO worsened the degree of intestinal apoptotic changes.

Conclusion: Results obtained in the study suggested that inhibition of iNOS, to some extent, improved intestinal architecture, while inhibition of nNOS failed to eliminate experimental intestinal heterophyiasis.
\end{abstract}

Keywords: intestinal heterophyiasis, iNOS, nitric oxide, nNOS, puppies.

Received: 11 February, 2021, Accepted: 5 April, 2021.

Corresponding Author: Marwa 0 Abdel Aziz, Tel.: +20 1114324769, E-mail: rycmarwa@gmail.com

Print ISSN: 1687-7942, Online ISSN: 2090-2646, Vol. 14, No. 1, April, 2021.

\begin{abstract}
Abbreviations: 7-NI: 7-Nitroindazole; AG: Aminoguanidine; ECM: Encysted metacercaria; IHC: Immunohistochemical; iNOS: Inducible nitric oxide synthase; NO: Nitric oxide; NOS: Nitric oxide synthase; nNOS: Neuronal nitric oxide synthase.
\end{abstract}

\section{INTRODUCTION}

Heterophyiasis is an intestinal illness caused by flukes belonging to the genus Heterophyes. The disease is endemic in many parts of the world, particularly the Nile Delta of Egypt and Southeast Asia ${ }^{[1,2]}$. Raw or inadequately cooked freshwater fish harboring viable EMC are the main source of human infection ${ }^{[3]}$. The disease typically presents with gastrointestinal symptoms that include mucoid diarrhea, abdominal colic, nausea and vomiting ${ }^{[4]}$. While asymptomatic infections are the most common, heavily infected cases are at the potential risk of extra-intestinal heterophyiasis. Ectopic lesions frequently involve heart, brain and spinal cord ${ }^{[5]}$. Crypt hyperplasia, villous atrophy and cellular infiltrations are among the main associated histopathological features. The infected intestinal mucosa may show blunting and edema of the villi, congestion, mastocytosis and goblet cell hyperplasia ${ }^{[6]}$. In experimental animal models infected with $H$. heterophyes, involvements of Peyer's patches and mesenteric lymph nodes were frequently seen $^{[7]}$.

The role of NO was reported among the immune mechanisms described in helminth infections ${ }^{[8]}$. It is known that NO is a free radical with different signaling functions in many cells $s^{[9]}$. It is produced by nitric oxide synthase (NOS) during the conversion of L-arginine to citrulline, with the participation of nicotinamide adenine dinucleotide phosphate as a cofactor. Three isoforms of NOS have been identified, which include inducible (iNOS), neuronal (nNOS) and endothelial (eNOS) ${ }^{[10]}$. It is known that iNOS is present in many cell types, including macrophages, neurons, and endothelial cells ${ }^{[11]}$. Whereas nNOS is mainly located in the central and peripheral nerves but has also been detected in non-neuronal cells, e.g. mast cells, epithelial cells, and neutrophils ${ }^{[12]}$. The 
eNOS was first identified in endothelial cells but is also present in smooth muscle cells, epithelial cells and $\mathrm{T}$ cells ${ }^{[13]}$. Both nNOS and eNOS are constitutively present in many cells and tissues where they participate in normal physiological responses. On the other hand, iNOS is an immune-inflammatory factor with critical signaling transduction events during infections ${ }^{[14]}$.

It is worth mentioning that the expression of NO contributes to regulating various processes in the gastrointestinal tract. The constitutive NOS enzymes have critical physiological functions such as maintaining motility and blood flow ${ }^{[15]}$. Additionally, iNOS acts as an anti-pathogen with tumoricidal activities. It is mainly involved in the defense mechanisms against infections and mediation of the inflammatory responses ${ }^{[16]}$. However, its over-production may cause detrimental consequences because of its cytotoxic activities ${ }^{[17]}$.

Despite the wide evidence of using iNOS due to its anti-parasitic effects in different experimental infections of trichinellosis ${ }^{[18]}$, fascioliasis ${ }^{[19]}$ and strongyloidiasis ${ }^{[20]}$, reports investigating the impact of nNOS in intestinal parasitic infections is lacking. Accordingly, we assessed, for the first time, the roles of iNOS and nNOS in intestinal heterophyiasis. After selective inhibition of both isoenzymes using 7-NI and $\mathrm{AG}$, we examined the possible consequences of such inhibition on experimentally infected puppies through parasitological, histopathological and IHC approaches.

\section{MATERIAL AND METHODS}

This experimental study was conducted at the Medical Parasitology Department (Post-Graduate Research Laboratory), Faculty of Medicine, Zagazig University, Zagazig, Egypt, during the period from July 2020 to December 2020.

Study design: EMC were collected to infect forty-four male puppies equally divided into four groups (11 puppies each). Control groups included non-infected puppies (G1) and infected untreated puppies (G2). Groups 3 and 4 included infected puppies treated with 7-NI and AG, respectively. Both drugs were administered after confirmation of the infection by detection of eggs in fecal samples. Drug treatment continued for 14 days post-infection. Three weeks post-infection, all puppies were sacrificed, and the whole intestines were resected, for further parasitological assessment, as well as histopathological and IHC evaluations.

Experimental animals: The puppies were of matched age (5 to 7 weeks old) and weight (3-3.5 kilograms). They were housed at the Parasitology Department, Faculty of Veterinary Medicine, Zagazig University, Zagazig, Egypt, in well-ventilated cages and fed bread and milk. Three consecutive fecal samples were examined to exclude any parasitic infections.
Collection of EMC and infection of puppies: One hundred Mugil cephalus and 200 Tilapia nilotica fish species were collected from local fish markets in Suez and Sharkia Governorates. They were placed in iceboxes, transferred to the Laboratory, and examined for the presence of EMC according to Mahdy et al. ${ }^{[21]}$. Detection of $H$. heterophyes EMC was based on their characteristic location between the muscle fibers, thick cyst wall and their relative large size ${ }^{[22]}$. Further microscopic identification was by the compression method $^{[23]}$. Detected EMC were separated from the infected fish samples using artificial digestion method $^{[24]}$. Only EMC with morphological characteristics compatible with those of $H$. heterophyes were used for infection. The final volume of EMC suspension was adjusted by saline to contain $\sim 300 / 0.5 \mathrm{ml}$ (the infective dose $)^{[25]}$. Infection of puppies was carried out orally using a stomach tube and mouth gage ${ }^{[26]}$.

To collect worms, the mucosa was gently scraped, and the contents were transferred to Petri dishes containing physiological saline. The collected worms were stained using the acetocarmine stain ${ }^{[27]}$ and were morphologically identified according to Witenberg ${ }^{[28]}$.

Drugs: Both 7-NI and AG drugs were obtained from Sigma-Aldrich (St Louis, MO, USA). The first was dissolved in dimethylsulfoxide (DMS) and made up to final volume by adding $0.9 \% \mathrm{NaCl}$ (DMS content was $15 \%)$. The first was given intraperitoneally at a dose of $30 \mathrm{mg} / \mathrm{kg} /$ day $^{[29]}$. The second was administered by intraperitoneal injection once daily $(100 \mathrm{mg} / \mathrm{kg})^{[30]}$.

Histopathological studies: The resected intestinal specimens from all study groups were fixed in 10\% formalin, dehydrated through grades of ethyl alcohol, cleared in xylol, and then processed for paraffin embedding. Serial cut sections of $5 \mu$ thickness were stained with haematoxylin and eosin (H\&E) $)^{[31]}$ Sectioned worms were studied concerning their site and the surrounding cellular infiltration. Intensity of infection was estimated by grading the sectioned worms per puppy. It was calculated in a semiquantitative manner according to Seo et al. ${ }^{[32]}$ as follows: score $0(-)$ : no worms; score $1(+)$ : less than 500; score $2(++)$ : 500 to less than 3000; and score $3(+++)$ : more than 3000 .

Other pathological changes such as intestinal polyps and apoptotic changes were also recorded. Apoptosis was graded as mean number of apoptotic cells $/ 10$ high power fields ${ }^{[33]}$ where $1=$ well-differentiated, $2=$ moderately differentiated, and $3=$ poorly differentiated; with corresponding mean numbers of apoptotic count/ high power fields of $0.86,2.21$, and 3.50 , respectively.

Immunohistochemical studies: The expressions of both iNOS and nNOS isoenzymes were evaluated using the avidin-biotin-peroxidase complex (ABC) method $^{[34]}$. Sections from paraffin blocks were dewaxed and antigens were retrieved in a microwave oven for 
20 min. For blocking intrinsic peroxidase activity $3 \%$ hydrogen peroxide solution was used. Primary antibody (rabbit anti-rat nNOS and iNOS antibody) (Beijing Zhongshan Biotechnology Co., Ltd, Beijing, China), in 1:200 dilution, was added and left overnight at $4^{\circ} \mathrm{C}$. Sections were then washed with phosphatebuffered saline and incubated with biotin-labelled goat anti-rabbit antibody for $35 \mathrm{~min}$, then again incubated with ABC kit, PK-4000 (Santa Cruz Biotechnology, Santa Cruz, CA, USA). Immunolabeling was attained by diaminobenzidine substrate which was added at room temperature. The slides were examined under light microscopy and the expression of the markers was recorded in a semiquantitative manner through optical density average by MetaMorph and computer image process software.

Statistical methods: The data were checked and analyzed using Statistical Package for the Social Sciences (SPSS) version 22. In the current study, nonparametric statistical methods were used ${ }^{[35]}$. MannWhitney (U) test was used as an alternative to the independent sample $t$-test which is used for comparing two groups ${ }^{[36]}$. The Wilcoxon signed-ranks test was used as the non-parametric alternative for the paired samples t- test ${ }^{[37]}$. Significance was considered when $P$ value was $<0.05$.

Ethical considerations: The dogs were reared and sacrificed according to the international guidelines approved by the Institutional Animal Care and Use of Zagazig University Committee (IACUC- ZU) for animal use in research and teaching.
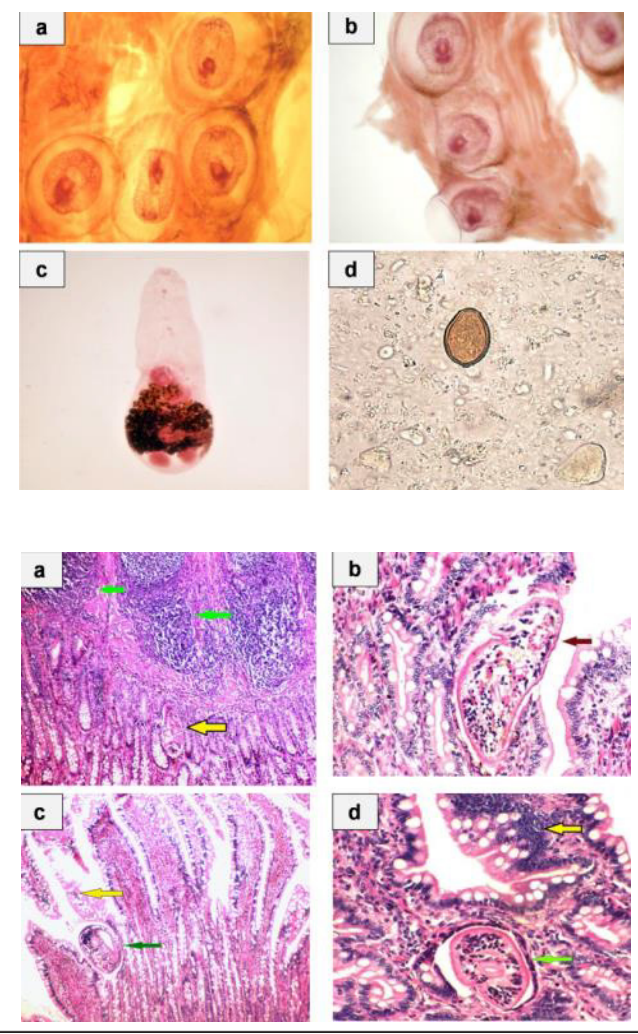

\section{RESULTS}

Parasitological results: Light microscopic examination of the fish samples revealed $H$. heterophyes EMC. They were found scattered in-between the striated muscle fibers and under the scales of the examined fish specimens (Fig. 1a, b). The majority of $H$. heterophyes adult worms (Fig. 1c) were collected from the upper portions of the small intestines. Few $H$. heterophyes eggs were recovered from the fecal samples of the infected animals one-week post-infection (Fig. 1d).

Intestinal sectioned worms: In G2, $H$. heterophyes adult worms were embedded in-between severely deformed and inflamed intestinal crypts and villi along with marked hyperplasia of lymphoid tissues (Fig. 2a, b). After administration of 7-NI in G3, worms were noticed between highly necrotic villi (Fig. 2c). In G4, AG significantly decreased the level of lymphocytic infiltration; however, it failed to eliminate the infection (Fig. 2d).

Intensity of infection: Figure (3) showed severe degrees of infection in $81.8 \%$ of both G2 and G4, and in $72.7 \%$ of G3. Also, both G2 and G3 (18.2\%), and G4 (9.1\%) displayed moderate degrees of infection. According to the obtained results and differences among the studied groups, severe worm scores of grade $3(>3000)$, and moderate scores of grade 2 (500$<3000$ ), were used to statistically compare reduction in intensity of infection between G2 and G3, and between G2 and G4, respectively. No significant reduction of intensity of infection $(P>0.05)$ was observed after
Fig. 1. Light microscopic examination showing: (a, b) $H$. heterophyes EMC in-between fish muscles (Aceto-carmine stain, X100); (c) H. heterophyes adult worm (Acetocarmine stain, X40); (d) H. heterophyes egg (X 400).
Fig. 2. Sectioned $H$. heterophyes worms in intestinal tissues of different study groups. Infected untreated group (G2) showed (a) $H$. heterophyes worm embedded in-between intestinal crypts (yellow arrow) and lymphoid tissue swelling (green arrows); (b) $H$. heterophyes adult between intestinal villi; (c) 7-NI treated group (G3) with the parasite (green arrow) between the necrotic villi (yellow arrow); (d) AG treated group (G4) with the parasite inbetween the crypts (green arrow) and reduction of inflammatory infiltration (yellow arrow) [H\&E stain, X 100 (a, c) X 400 (b, d)]. 
administration of either 7-NI (G3) or AG (G4) when compared to infected untreated puppies (G2) (Table 1).

Histopathological changes: Normal features of the small intestinal sections were observed in G1 (Fig. $4 \mathrm{a}$ ), as compared to sections of G2, in which there is a characteristic loss of the normal crypt architecture and marked lymphocytic inflammatory infiltrations (Fig. 4b). Other pathological mucosal changes were also recorded in G2 such as goblet cell loss and villous blunting (Fig. 4c, d). Following the administration of 7-NI in G3, a notable loss of the intestinal tissue architecture was demonstrated (Fig. 5a). The degrees of both lymphocytic and eosinophilic inflammatory cellular infiltrations were markedly increased (Fig. 5 a, b). Other pathological changes were also detected such as apoptotic changes (Fig. 5c) and intestinal polyp (Fig. $5 d)$.

According to the obtained results, administration of 7-NI in G3 significantly worsened the degrees of apoptosis $(P<0.001)$, when compared to the infected untreated group (G2). No significant increase in the apoptotic grades $(P>0.05)$ was observed after the administration of $\mathrm{AG}$ in $\mathrm{G} 4$ when compared to $\mathrm{G} 2$ (Table 2).

Despite the persistence of erosions in the intestinal sections of G4 (Fig. 6a), the degree of inflammatory infiltration remarkably decreased (Fig. 6b), with a

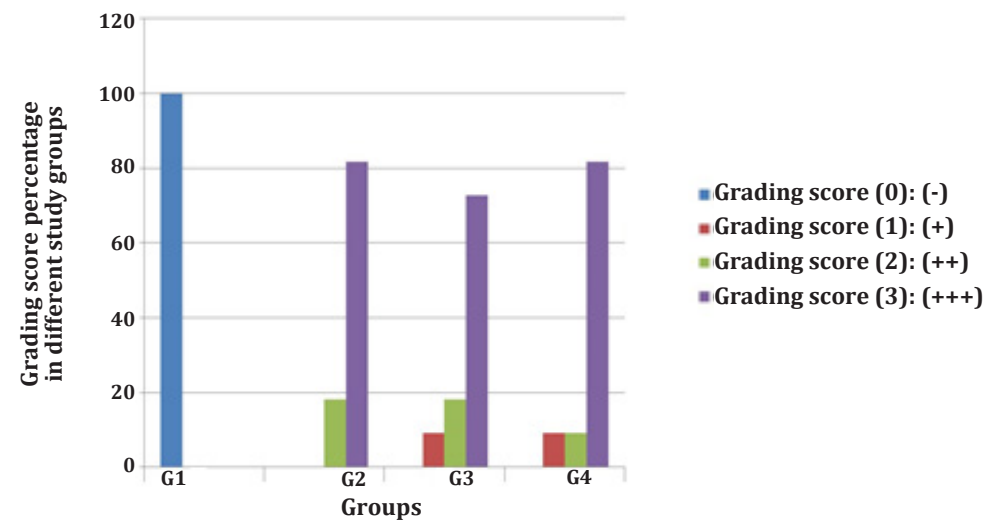

Fig. 3. Grading of the sectioned $H$. heterophyes worms in intestinal tissues of different study groups. (-): no worms; Score 1 (+): less than 500; Score 2 (++): 500 to less than 3000; Score 3 (+++): more than 3000.

Table 1. Comparison of grading scores of worm's infections between the studied groups.

\begin{tabular}{llccccc}
\hline \hline Grade & Group & No. & Infection\% & Mean rank & Sum of ranks & Statistical analysis $^{\circledR}$ \\
\hline \multirow{2}{*}{$\mathbf{3}$} & G2 & 11 & 81.8 & 11.54 & 761.50 & $U=333.00, Z=-0.143$ \\
& G3 & 11 & 72.7 & 10.63 & 701.50 & $P>0.050$ (NS) \\
\hline \multirow{2}{*}{$\mathbf{2}$} & G2 & 11 & 18.2 & 11.63 & 767.50 & $U=327.00, Z=-0.172$ \\
& G4 & 11 & 9.1 & 10.54 & 595.50 & $P>0.050$ (NS) \\
\hline \hline
\end{tabular}

Grading score 2: 500-<3000 worms; Grading score 3: More than 3000 worms; G2: Infected untreated group; G3: Infected 7-NI treated group; G4: Infected AG treated group; No.: Number of puppies in each group; @: Mann-Whitney test; NS: Non-significant $(P>0.05)$.

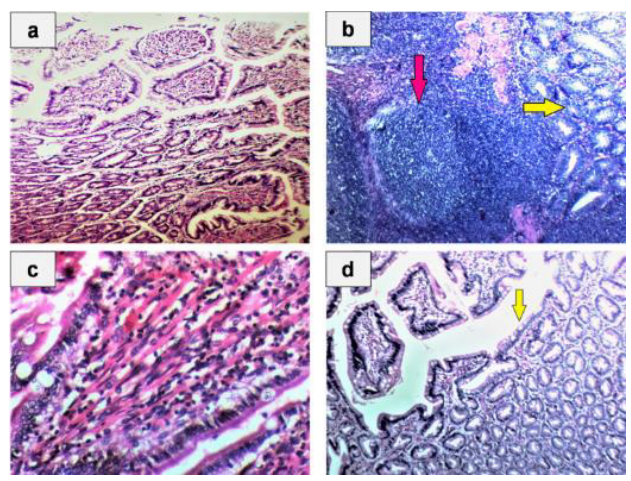

Fig. 4. Histopathological intestinal features of G1 and G2 (a): Control non-infected group (G1) showed normal tissue architecture. (b-d): Infected non-treated group (G2) showed (b) marked lymphocytic infiltration (red arrow) with loss of normal crypt structure (yellow arrow); (c) marked goblet cell loss; (d) severe villous blunting (yellow arrow) [H\&E stain, $\mathrm{X}$ $100(\mathrm{a}, \mathrm{d})$, and X $400(\mathrm{~b}, \mathrm{c})]$.

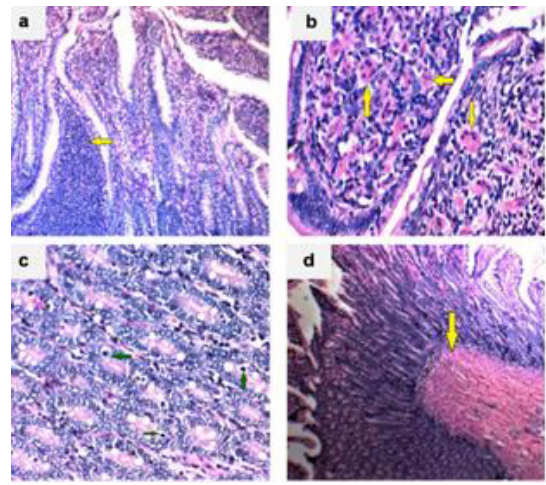

Fig. 5. Histopathological intestinal features of 7-NI treated puppies (G3) showed (a) loss of normal tissue architecture with severe lymphocytic infiltration (yellow arrow); (b) marked eosinophilic cellular infiltrations (yellow arrows); (c) distinctive apoptotic changes (green arrows); (d) intestinal polyp (yellow arrow) [H\&E stain, X 100 (a ,d) and $\mathrm{X} 400(\mathrm{~b}, \mathrm{c})]$. 
statistically significant difference $(P<0.001)$ compared to G2 (Table 3). There was also a notable recovery of goblet cells (Fig. 6c). Moreover, intestinal architecture was nearly restored to normal (Fig. 6d).

Table 2. Comparison of grading scores of apoptotic changes between the studied groups.

\begin{tabular}{|c|c|c|c|c|c|c|}
\hline Grade & Group & No. & Infection $\%$ & Mean rank & Sum of ranks & Statistical analysis $^{@}$ \\
\hline \multirow{2}{*}{1} & G2 & 11 & 36.4 & 16.27 & 179.00 & \multirow{2}{*}{$\begin{aligned} U= & 8.00, Z=3.560 \\
& \boldsymbol{P}<\mathbf{0 . 0 0 1}\end{aligned}$} \\
\hline & G3 & 11 & 18.2 & 6.73 & 74.00 & \\
\hline \multirow{2}{*}{3} & G2 & 11 & 18.2 & 6.00 & 66.00 & \multirow{2}{*}{$\begin{aligned} U= & 0.00, Z=4.421 \\
& P<\mathbf{0 . 0 0 1}\end{aligned}$} \\
\hline & G3 & 11 & 45.5 & 17.00 & 187.00 & \\
\hline \multirow{2}{*}{3} & G2 & 11 & 18.2 & 10.13 & 668.50 & \multirow{2}{*}{$\begin{array}{c}U=300.00, Z=-0.29 \\
P>0.05(\mathrm{NS})\end{array}$} \\
\hline & G4 & 11 & 27.3 & 12.04 & 794.50 & \\
\hline
\end{tabular}

Grade 1: Well-differentiated cells (0.86); Grade 3: Poorly differentiated cells (3.5); G2: Infected untreated group; G3: Infected 7-NI treated group; G4: Infected AG treated group; No.: Number of puppies in each group; @: Mann-Whitney test; NS: Non-significant $(P>0.05)$; *: Significant $(P<0.001)$.

Table 3. Comparison between degrees of inflammatory infiltration changes in G2 and G4.

\begin{tabular}{lcccc}
\hline \hline Group & No. & $\begin{array}{c}\text { Mean } \\
\text { rank }\end{array}$ & $\begin{array}{c}\text { Sum of } \\
\text { ranks }\end{array}$ & Statistical analysis \\
\hline G2 & 11 & 14.45 & 953.50 & $U=141.00, Z=-1.032$ \\
G4 & 11 & 7.72 & 509.50 & $\boldsymbol{P}<\mathbf{0 . 0 0 1}{ }^{*}$ \\
\hline
\end{tabular}

G2: Infected untreated group; G4: Infected AG treated group; No.: Number of puppies in each group; @: MannWhitney test; *: Significant $(P<0.001)$.

Immunohistochemical results: Both iNOS and nNOS isoenzymes were observed with variable densities in the intestinal sections of different study groups. The highest concentration of iNOS isoenzyme was detected in G3 after administration of 7-NI. While the highest detection of nNOS isoenzyme was demonstrated in the control non-infected group (G1). There was a significant difference $(P<0.05)$ between the distribution of the two markers in both G1 and G3. On the other hand, there was no significant difference between the distribution of the markers in G2 or G4 (Table 4).

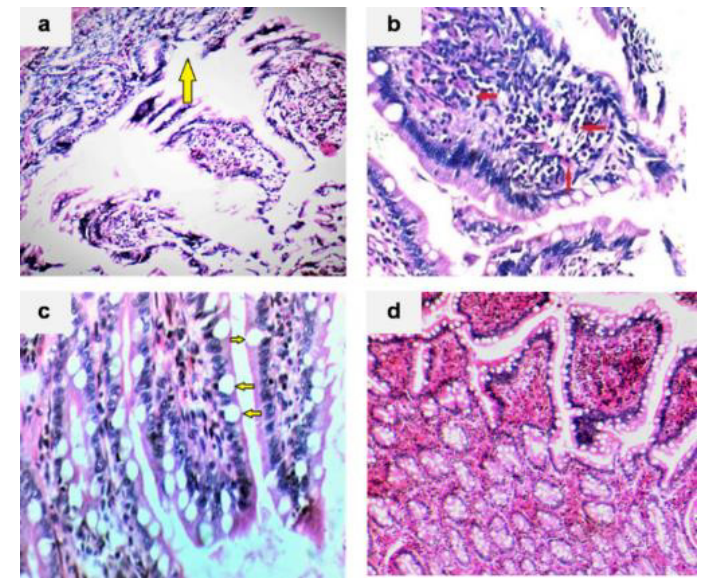

Fig. 6. Histopathological intestinal features of AG treated puppies (G4), showed (a) intestinal erosion (yellow arrow); (b) remarkable reduction of eosinophilic cellular infiltrations (red arrows); (c) recovery of goblet cells (yellow arrows); (d) restoration of normal architecture [H\&E stain, X 400 (ac) $\mathrm{X} 100(\mathrm{~d})]$.

Table 4. Distribution of nNOS and iNOS markers in the intestinal sections of the studied groups.

\begin{tabular}{|c|c|c|c|c|c|}
\hline Group & Ranks & No. & Mean rank & Sum of ranks & Statistical analysis $^{@}$ \\
\hline \multirow{4}{*}{ G1 } & Negative ranks & 2 & 2.58 & 31.00 & \multirow{4}{*}{$\begin{array}{c}Z=-0.415 \\
P<0.05^{*}\end{array}$} \\
\hline & Positive ranks & 4 & 3.33 & 80.00 & \\
\hline & Ties & 5 & 0.00 & 0.00 & \\
\hline & Total & 11 & ---- & ---- & \\
\hline \multirow{4}{*}{ G2 } & Negative ranks & 1 & 1.00 & 1.00 & \multirow{4}{*}{$\begin{array}{c}Z=-1.000 \\
P>0.05(\mathrm{NS})\end{array}$} \\
\hline & Positive ranks & 0 & 0.00 & 0.00 & \\
\hline & Ties & 10 & 0.00 & 0.00 & \\
\hline & Total & 11 & $-\cdots--$ & $-\cdots--$ & \\
\hline \multirow{4}{*}{ G3 } & Negative ranks & 7 & 4.00 & 28.00 & \multirow{4}{*}{$\begin{array}{c}Z=-2.530 \\
P<\mathbf{0 . 0 5}^{*}\end{array}$} \\
\hline & Positive ranks & 0 & 0.00 & 0.00 & \\
\hline & Ties & 4 & 0.00 & 0.00 & \\
\hline & Total & 11 & ---- & ---- & \\
\hline \multirow{4}{*}{ G4 } & Negative ranks & 2 & 1.50 & 3.00 & \multirow{4}{*}{$\begin{array}{c}Z=-1.342 \\
P>0.05(\mathrm{NS})\end{array}$} \\
\hline & Positive ranks & 0 & 0.00 & 0.00 & \\
\hline & Ties & 9 & 0.00 & 0.00 & \\
\hline & Total & 11 & ---- & ----- & \\
\hline \multicolumn{6}{|c|}{$\begin{array}{l}\text { G1: Control non-infected group; G2: Infected untreated group; G3: Infected-7-NI treated group; G4: Infected-AG treated group; } \\
\text { nNOS: Neuronal nitric oxide synthase; iNOS: Inducible nitric oxide synthase; Negative rank: Intestinal nNOS < intestinal iNOS; } \\
\text { Positive rank: Intestinal nNOS > intestinal iNOS; Ties: Intestinal nNOS = intestinal iNOS; *: Significant }(P<0.05) \text {; NS: Non-significant } \\
(P>0.05) \text {; @: Wilcoxon signed ranks test. }\end{array}$} \\
\hline
\end{tabular}


Isoenzymes expression in intestinal sections of different study groups: Control non-infected dogs (G1) showed negative expression of the iNOS isoenzyme (Fig. 7a). After $H$. heterophyes infection in G2, moderate iNOS concentration was detected (Fig. 7b). It was observed that 7-NI caused a notable increase in the marker expression in G3 (Fig. 7c). On the other hand, the intestinal sections of G4 exhibited a
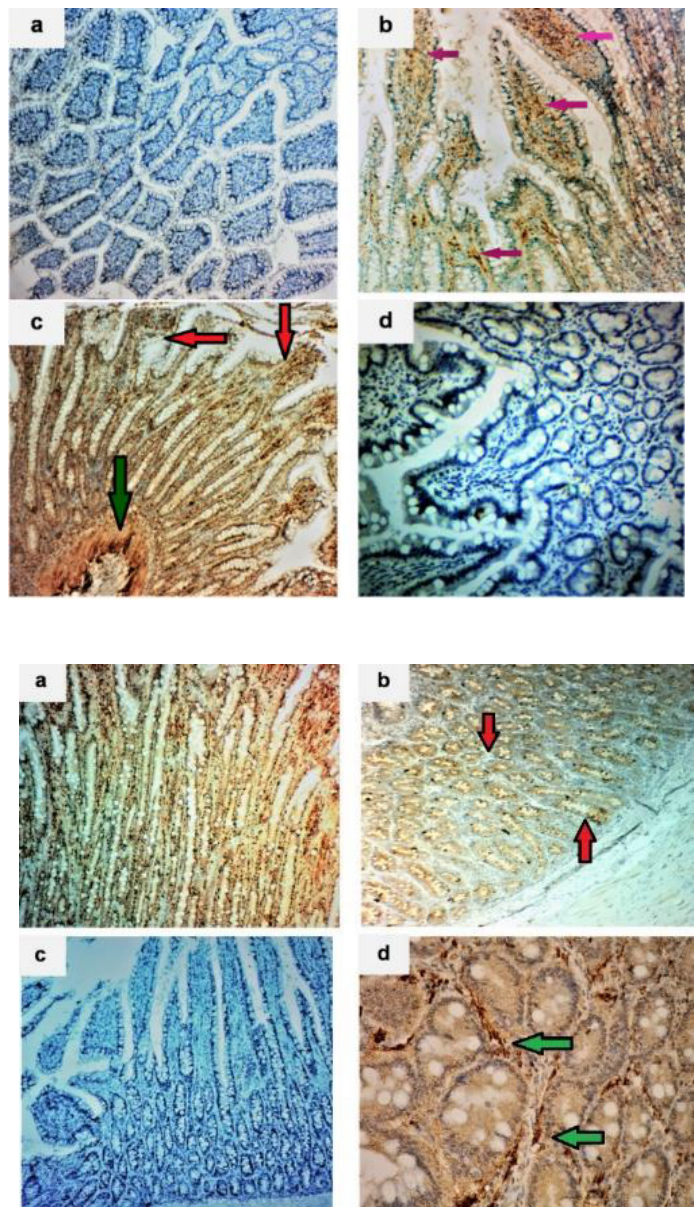

\section{DISCUSSION}

The effective immune response against helminth infection is mainly a Th2- mediated response. Yet, various studies have shown that the release of NO may have a vital role in the elimination of extracellular parasites such as, B. malayi $^{[38]}, A$. suum ${ }^{[39]}$ and $E$. histolytica $^{[40]}$. The functional roles of NO and NOS isoforms in the anti-parasitic immune response, especially in heterophyiasis, is not yet completely understood. In this respect, this study aimed to explore the possible effects of iNOS and nNOS in experimental intestinal heterophyiasis. Puppies were chosen as relatively large animal models for infection because the innervation of their intestinal tract is similar to that of humans ${ }^{[41]}$.

The degree of pathological changes in heterophyiasis is apparently related to the intensity of the infection ${ }^{[22]}$. In the current study, $81.8 \%$ of $H$. heterophyes infected puppies in G2 exhibited a severe degree of the infection negative expression of the marker (Fig. 7d). Concerning nNOS, high levels were demonstrated in the intestinal sections of the control non-infected group (G1) (Fig. 8a). Strong expression of the marker was also reported in the intestinal glands of G2 (Fig. 8b). Negative nNOS expression was detected in the intestinal sections of G3 (Fig. 8c). In G4, AG induced a notable reduction in the nNOS enzyme expression (Fig. 8d).

Fig. 7. Immunohistochemical expression of iNOS in the intestinal sections of different study groups showed (a) negative expression in control non-infected group (G1); (b) moderate expression in infected non-treated group (G2) (brown coloration, pink arrows); (c) strong expression inside the villi (brown coloration, red arrows) and inside lamina propria (brown coloration, green arrow) in 7-NI treated group (G3); (d) negative expression in AG treated group (G4) (IHC stain, $X$ 400).

Fig. 8. Immunohistochemical expression of nNOS in the intestinal sections of different study groups showed (a) control non-infected group (G1) with high density of nNOS infiltration (brown coloration); (b) infected non-treated group (G2) with strong marker expression in the intestinal glands (brown coloration, red arrows); (c) 7-NI treated group (G3) with negative marker expression; (d) AG treated group (G4) with weak nNOS expression in-between intestinal glands (brown coloration, green arrows) [IHC stain, X 100 (a-c) X400 (d)].

(Fig. 3), which resulted in several pathological events: marked lymphocytic inflammatory infiltration, loss of normal intestinal tissue architecture, goblet cell loss and villous blunting (Fig. 4b-d). The recorded pathological impacts are in accordance with those reported by Daoud et al. ${ }^{[41]}$. Also, Shams-Eldin ${ }^{[42]}$ reported inflammatory cellular infiltrations, congestion and fibrosis in the intestinal tissue sections of dogs experimentally infected with $H$. heterophyes.

Inhibition of nNOS and the subsequent upregulation of iNOS after 7-NI administration in G3 failed to eliminate the infection, where $72.7 \%$ exhibited severe degrees of the infection (Fig. 3). In support, Li et al. ${ }^{[43]}$ observed that while NO production by iNOS is not necessary for $G$. lamblia elimination, nNOS played a vital role in the elimination of giardiasis. Therefore, the pathological changes recorded in G3 could be attributed to increased expression of iNOS enzyme and reduced nNOS levels. These pathological changes included increased lymphocytic and eosinophilic 
inflammatory infiltration, distinctive apoptotic changes as well as intestinal polyp formation (Fig. 5). According to table (2), marked degrees of apoptotic changes were reported in G3 compared to G2, with a statistically significant difference $(P<0.001)$. The harmful effects of increased iNOS isoenzyme expression on the integrity of the intestinal mucosa were also recorded by Lu et al. ${ }^{[44]}$ who demonstrated a significant correlation between intestinal injury in neonatal rats due to necrotizing enterocolitis and iNOS increased expression.

The degree of cellular inflammatory infiltration was significantly reduced $(P<0.001)$ after $A G$ administration when compared to the infected untreated group (G2) (Table 3). Tahir et al. ${ }^{[45]}$ confirmed the antiinflammatory effect of $A G$ as a selective inhibitor of iNOS. Moreover, Salvemini et al. ${ }^{[46]}$ demonstrated the involvement of NO in the development of inflammation. In the present study, inhibition of NO by AG resulted in significant goblet cell recovery (Fig. 6c). Weidinger et $a{ }^{\left[{ }^{[47]}\right.}$ substantiated the key role of NO in up-regulating goblet cells and increasing mucous secretion. Despite the persistence of parasitic infection in G4, following the administration of $\mathrm{AG}$, the intestinal tissue architecture was nearly restored to normal (Fig. 6d). Also, Malinski ${ }^{[48]}$ described the toxic impacts of increased levels of NO on healthy tissues.

In the current study, IHC labelling of iNOS and nNOS isoenzymes was demonstrated in the intestinal sections of different groups. Control non-infected puppies (G1) showed a negative expression of iNOS isoenzyme (Fig. $7 a$ ), but with high concentration of nNOS isoenzyme (Fig. $8 a$ ). The difference was statistically significant (Table $4, P<0.05)$. These findings agree with those reported by Takahashi ${ }^{[49]}$ who denoted that iNOS is not usually present in tissues under physiological conditions, but is induced during tissue injury and inflammation. Also, $\mathrm{Qu}$ et $a l^{\left[{ }^{[50]}\right.}$ reported that nNOS is the predominant isoenzyme form in rat small intestine and accounts for more than $90 \%$ of the total NO synthases.

In the infected untreated control G2, heterophyiasis induced a moderate increase in iNOS expression (Fig. $7 b)$. On the other hand, nNOS was strongly expressed in the intestinal glands of G2 (Fig. 8b). Babu and Griffith ${ }^{[51]}$ reported conditions, other than infections, which could cause an up-regulation in the nNOS enzyme level such as neurodegenerative disorders, pain and ischemia.

Following the administration of 7-NI in G3, a marked increase in iNOS expression was detected (Fig. 7c); coinciding with negative nNOS enzyme expression in the intestinal sections (Fig. 8c). Such a difference in the concentration between both markers was statistically significant (Table $4, P<0.05$ ). The selective inhibitory effect of 7-NI on the intestinal nNOS enzyme expression was also reported ${ }^{[52]}$. The researchers showed the antiinflammatory effect of nNOS isoenzyme on preserving the morphological intestinal integrity of experimentally ischaemic dogs when using 7-NI drug as a selective inhibitor of nNOS. Also, the reported up-regulation of iNOS levels induced by 7-NI was described ${ }^{[44]}$. Qu et $a l^{[53]}$ explained the mechanism of nNOS in regulating intestinal levels of iNOS enzyme through modulation of nuclear factor kappa B.

Negative iNOS enzyme expression was demonstrated in the intestinal sections of AG treated G4 puppies (Fig. 7d). The suppressive effect of AG on iNOS enzyme was reported by Misko et al. ${ }^{[54]}$ who denoted the value of $A G$ in reducing a variety of inflammatory and immunologically mediated diseases through its inhibition of the nitric oxide overproduction. Additionally, AG caused a reduction in the nNOS expression in the intestinal sections of G4 (Fig. 8d). Jianmongkol et al. ${ }^{[55]}$ and Alderton et al. ${ }^{[56]}$ demonstrated that AG inhibits all isoforms of NOS, with iNOS being much more sensitive than nNOS or eNOS. Whereas Jeyabal et al. ${ }^{[57]}$ demonstrated the beneficial role of $A G$ in preventing the reduction of duodenal nNOS expression in diabetic rats.

There were two potential limitations in our evaluation of NO possible roles in the immune response of puppies to heterophyiasis. First, the study should have included two additional control non-infected puppies treated by 7-NI or AG to show NO role in the normal immune response of puppies. Using noninfected puppies treated with selective inhibitor of iNOS may explain the apoptotic changes due to drug administration under normal conditions. On the other hand, using non-infected puppies treated with selective inhibitor of nNOS may explain its failure to eliminate heterophyiasis. Second, there may have been a notable reduction in severity of apoptic changes if the infected puppies were sacrificed at an earlier time, less than 3 weeks post-infection, or if AG was administered for only one week.

To conclude, our study showed that heterophyiasis induces an increase in iNOS expression, but with no effect on nNOS levels. The study also indicated that over-production of iNOS by administration of 7-NI exacerbated the pathological deficits in the small intestinal sections of the infected puppies, in contrast to the deficient iNOS by AG treatment. Selective blockage of nNOS failed to clear the infection. Further experiments are recommended to elucidate the role of nNOS in the elimination of different intestinal parasites.

Acknowledgement: The authors are grateful to Prof. Hayam H. Rashed, Pathology Department, Faculty of Medicine, Zagazig University, for her assistance in the histopathological and IHC studies.

Author contributions: Abdel Aziz MO conceptualized the idea, and wrote the first draft of the manuscript. Elsayed HO conducted the statistical analysis. Both authors performed the study design and all experimental studies, and contributed in all previous 
versions of the manuscript. Authors have also read and approved the final manuscript.

Conflicts of interest: The authors report no conflicts of interest regarding the publication of this manuscript.

Funding statement: This research did not receive any specific grants from funding agencies in the public, commercial or not-for-profit sectors.

\section{REFERENCES}

1. Simões SB, Barbosa HS, Santos CP. The life cycle of Ascocotyle (Phagicola) longa (Digenea: Heterophyidae), a causative agent of fish-borne trematodosis. Acta Trop 2010; 113(3):226-233.

2. Graczyk TK, Fried B. Human waterborne trematode and protozoan infections. Adv Parasitol 2007; 64:111-160.

3. Pica R, Castellano C, Cilia C, Errico FP. Intestinal fluke infections: the heterophyids. Clin Ter 2003; 15(1):6163.

4. Toledo R, Esteban JG, Fried B. Immunology and pathology of intestinal trematodes in their definitive hosts. Adv Parasitol 2006; 63:285-365.

5. Zhang SL, Fan GH. Case report of brain abscess caused by heterophyid eggs. Chin J Parasitol Parasitic Dis 1990; 8:178-183.

6. Chai JY, Lee SH. Food-borne intestinal trematode infections in the Republic of Korea. Parasitol Int 2002; 51:129-154.

7. Hamdy EI, Nicola E. On the histopathology of the small intestine in animals experimentally infected with Heterophyes heterophyes. J Egypt Med Assoc 1980; 63: 179-184.

8. Pfarr KM, Qazi S, Fuhrman JA. Nitric oxide synthase in filariae: demonstration of nitric oxide production by embryos in Brugia malayi and Acanthocheilonema viteae. Exp Parasitol 2001; 97:205-214.

9. Baranano DE, Ferris CD, Snyder SH. Atypical neural messengers. Trends Neurosci 2001; 24:99-106.

10. Paakkari I, Lindsberg P. Nitric oxide in the central nervous system. Ann Med 1995; 27:369-377.

11. Bogdan C, Rollinghoff M, Diefenbach A. Reactive oxygen and reactive nitrogen intermediates in innate and specific immunity. Curr Opin Immunol 2000; 12:64-76.

12. Asano K, Chee CB, Gaston B, Lilly CM, Gerard C, Drazen $\mathrm{JM}$, et al. Constitutive and inducible nitric oxide synthase gene expression, regulation, and activity in human lung epithelial cells. Proc Natl Acad Sci USA 1994; 91:10089-10093.

13. Forstermann U, Boissel JP, Kleinert H. Expressional control of the 'constitutive' isoforms of nitric oxide synthase (NOS I and NOS III). FASEB J 1998; 12:773790.

14. Xie QW, Cho HJ, Calaycay J, Mumford RA, Swiderek KM, Lee TD, et al. Cloning and characterization of inducible nitric oxide synthase from mouse macrophages. Science 1992; 10:256(5054):225-228.

15. Miller MJ, Zhang XJ, Sadowska-Krowicka $H$, Chotinaruemol S, McIntyre JA, Clark DA, et al. Nitric oxide release in response to gut injury. Scand Gastroenterol 1993; 28:149-154.

16. Nathan C. Inducible nitric oxide synthase: what difference does it make? J Clin Invest 1997; 100: 2417-2423.

17. Gradoni L, Ascenzi P. Nitric oxide and anti-protozoan chemotherapy. Parassitologia 2004; 46(1-2):101103.

18. Zeromski J, Boczoń K, Wandurska-Nowak E, Mozer-Lisewska I. Effect of aminoguanidine and albendazole on inducible nitric oxide synthase (iNOS) activity in Trichinella spiralis-infected mice muscles. Folia Histochem Cytobiol 2005; 43(3): 157:159.

19. Beytut E, Akca A, Gokce HI. Pathological and immunohistochemical evaluation of the effects of interferon gamma (IFN- $\gamma$ ) and aminoguanidine in rats experimentally infected with Fasciola hepatica. Turk J Vet Anim Sci 2011; 4:243-253.

20. Rodrigues RM, Gonçalves ALR, Silva NM, Cardoso CRB, Araújo NR, Coutinho LB, et al. Inducible nitric oxide synthase controls experimental Strongyloides infection. Parasite Immunol 2018; 40(10):e12576.

21. Mahdy OA, Essa MA, Easa MES. Parasitological and pathological studies in Tilapia sp. from Manzala Egypt. J Comp Pathol Clin Pathol 1995; 8:131-145.

22. Sohn WM. Fish-borne zoonotic trematode metacercariae in the Republic of Korea. Korean J Parasitol 2009; 47:103-116.

23. El-Sheikha HM, El-Shazly AM. Host-dependent variations in the seasonal prevalence and intensity of heterophyid encysted metacercariae (Digenea: Heterophyidea) in brackish water fish in Egypt. Vet Parasitol 2008; 153:65-72.

24. Yokogawi M, Sano M. Studies on the intestinal flukes IV. On the development of the worm in the experimental infected animals with metacercariae of Metagonimus yokogawi. Japan Parasitol 1968; 17:540-545.

25. Ashour DS, Othman AA, Radi DA. Insights into regulatory molecules of intestinal epithelial cell turnover during experimental infection by Heterophyes heterophyes. Exp Parasitol 2014;143:48-54.

26. Hegazi MA, Hassan AT, Al-Nashar TM, AboElkheir OI, El-Lessi FM. Encysted metacercariae of family Heterophyidae in infected fish in Dakahlia Governorate, an endemic focus in Egypt. J Egypt Soc Parasitol 2014; 44(3):547-558.

27. Cheesbrough M. Medical Laboratory Manual for Tropical Countries, $2^{\text {nd }}$ ed. 1987; ELBS Tropical Health Technology; Butterworth.

28. Witenberg G. Studies on the trematode family Heterophyidae. Ann Trop Med Parasitol 1929; 23 (2):131-139.

29. Luo CX, Zhu XJ, Zhou QG, Wang B, Wang W, Cai $\mathrm{HH}$, et al. Reduced neuronal nitric oxide synthase is involved in ischemia-induced hippocampal neurogenesis by up-regulating inducible nitric 
oxide synthase expression. J Neurochem 2007; 103(5):1872-1882.

30. Khan IA, Schwartzman JD, Matsuura T, Kasper LH. A dichotomous role for nitric oxide during acute Toxoplasma gondii infection in mice. Proc Natl Acad Sci USA 1997; 94(25):13955-13960.

31. Drury RAB, Wallington EA. Carleton's histological technique, $5^{\text {th }}$ ed. 1980; Oxford University Press, Oxford, New York, Toronto.

32. Seo BS, Cheong SK, Chai JY, Lee SH, Lee JB. Histopathology of small intestines of rats and mice experimentally infected with Pygidiopsis summa. Seoul J Med 1986; 2:125-134.

33. Seth A, Agarwal A. Apoptotic count as a guide for histological grading of carcinoma esophagus: a light microscopic study. J Lab Physicians 2009; 1(1):11-14.

34. Czarnewski P, Araujo ECB, Oliveira MC, Mineo TWP, Silva NM. Recombinant TgHSP70 immunization protects against Toxoplasma gondii brain cyst formation by enhancing inducible nitric oxide expression. Front Cell Infect Microbiol 2017; 7:142.

35. Whitley E, Ball J. Statistics review 6: Nonparametric methods. Crit Care 2002; 6:509-513.

36. Chan YH. Biostatistics 102: Quantitative dataparametric and non-parametric tests. Singap Med J 2003; 44(8):391-396.

37. Mantas J. Statistical methods. Stud Health Technol Inform 2002; 65:136-147.

38. Rajan TV, Porte P, Yates JA, Keefer L, Shultz LD. Role of nitric oxide in host defense against an extracellular, metazoan parasite Brugia malayi. Infect Immun 1996; 64:3351-3353.

39. Bascal ZA, Cunningham JM, Holden-Dye L, O'Shea M, Walker RJ. Characterization of a putative nitric oxide synthase in the neuromuscular system of the parasitic nematode Ascaris suum. Parasitology 2001; 122: 219-231.

40. Hernández-Campos ME, Campos-Rodríguez R, Tsutsumi V, Shibayama M, García-Latorre E, CastilloHenkel C, et al. Nitric oxide synthase in Entamoeba histolytica: its effect on rat aortic rings. Exp Parasitol 2003; 104(3-4):87-95.

41. Daoud A, El-Kowrany S, Deyab F, Eliwa K. Experimental heterophyiasis: Histopathological and immunological study. Int Med J 2012; 42:7.

42. Shams-Eldin S. Histopathological, histochemical and immunohistochemical studies of experimental heterophyiasis in dogs and the protecting role of praziquantel. PUJ 2011; 4(2):185-192.

43. Li E, Zhou P, Singer SM. Neuronal nitric oxide synthase is necessary for elimination of Giardia lamblia infections in mice. J Immunol 2006; 176(1):516-521.

44. Lu H, Zhu B, Xue XD. Role of neuronal nitric oxide synthase and inducible nitric oxide synthase in intestinal injury in neonatal rats. World J Gastroenterol 2006; 12(27):4364-4368.
45. Tahir S, Badshah A, Hussain RA. Guanidines from 'toxic substances' to compounds with multiple biological applications: Detailed outlook on synthetic procedures employed for the synthesis of guanidines. Bioorg Chem 2015; (59):39-79.

46. Salvemini D, Wang ZQ, Wyatt PS, Bourdon DM, Marino $\mathrm{MH}$, Manning PT, et al. Nitric oxide: A key mediator in the early and late phase of carrageenan-induced rat paw inflammation. Br J Pharmacol 1996; 4:829-838.

47. Weidinger A, Müllebner A, Paier-Pourani J, Banerjee A, Miller I, Lauterböck L, et al. Vicious inducible nitric oxide synthase-mitochondrial reactive oxygen species cycle accelerates inflammatory response and causes liver injury in rats. Antioxid. Redox Signal 2015; 7:572-586.

48. Malinski T. Using nano-sensors for in situ monitoring and measurement of nitric oxide and peroxynitrite in a single cell. Methods Mol Biol 2015; 1208:139-155.

49. Takahashi T. Pathophysiological significance of neuronal nitric oxide synthase in the gastrointestinal tract. J Gastroenterol 2003; 38(5):421-430.

50. Qu XW, Wang H, Rozenfeld RA, Huang W, Hsueh W. Type I nitric oxide synthase (NOS) is the predominant NOS in rat small intestine: Regulation by PAF. Biochim Biophys Acta 1999; 1451:211-217.

51. Babu BR, Griffith OW. Design of isoform-selective inhibitors of nitric oxide synthase. Curr Opin Chem Biol 1998; 2:491-500.

52. Varga S, Juhász L, Gál $\mathrm{P}$, Bogáts G, Boros $\mathrm{M}$, Palásthy $\mathrm{Z}$, et al. Neuronal nitric oxide mediates the anti-inflammatory effects of intestinal ischemic preconditioning. J Surg Res 2019; 244:241-250.

53. Qu XW, Wang H, De Plaen IG, Rozenfeld RA, Hsueh W. Neuronal nitric oxide synthase (NOS) regulates the expression of inducible NOS in rat small intestine via modulation of nuclear factor kappa B. FASEB J 2001; 15(2):439-446.

54. Misko TP, Moore WM, Kasten TP, Nickols GA, Corbett JA, Tilton RG, et al. Selective inhibition of the inducible nitric oxide synthase by aminoguanidine. Eur J Pharmacol 1993; 233(1):119-125.

55. Jianmongkol S, Vuletich JL, Bender AT, Demady DR, Osawa Y. Aminoguanidine-mediated inactivation and alteration of neuronal nitric-oxide synthase. J Biol Chem 2000; 275:13370-13376.

56. Alderton WK, Cooper CE, Knowles RG. Nitric oxide synthases: structure, function and inhibition. Biochem J 2001; 357:593-615.

57. Jeyabal PV, Kumar R, Gangula PR, Micci MA, Pasricha PJ. Inhibitors of advanced glycation end-products prevent loss of enteric neuronal nitric oxide synthase in diabetic rats. Neurogastroenterol Motil 2008; 20(3):253-261. 